\section{Série temporal de características maternas e de nascidos vivos em Niterói, RJ}

\section{Time serie of characteristics of mothers and live newborns in Niterói, $R J$}

Suelem do Rozario 1

Alexandre dos Santos Brito 2

Pauline Lorena Kale 3

Sandra Costa Fonseca 4
1,4 Instituto de Saúde da Comunidade. Universidade Federal Fluminense. Rua Marquês de Paraná, 303. $3^{\circ}$ andar, sala 6. Niterói, RJ, Brasil. CEP: 24.030-215. E-mail: sandracfonseca@yahoo.com.br

2,3 Instituto de Estudos em Saúde Coletiva. Universidade Federal do Rio de Janeiro. Rio de Janeiro, RJ, Brasil.

\section{Abstract}

Objectives: to describe the tendency over time of characteristics of the mother, care provided and newborns, in Niterói, a city in the Brazilian State of Rio de Janeiro.

Methods: an ecological time-series study from 2000 to 2009. Data were gathered from the Live Birth Information System (SINASC). Annual variations were described in age, schooling and reproductive history of mother, prenatal care, kind of delivery, color, birth weight and gestational age.

Results: the completeness of information was high among the 62,449 live newborns studied. Births fell and primiparity increased. There was reduction in pregnancies among adolescents (2.3\% per year) and an increase in mothers aged over 35 years. Schooling improved, with a decrease in the percentage of pregnant women with less than eight years of school. Around $80 \%$ of women had attended seven or more prenatal sessions. Caesarean births tended to increase in frequency, with $67.7 \%$ of woman having one of these in 2009. Low birth weight showed a tendency to fall by $1.1 \%$ per year. Prematurity increased by 7.4 to $7.9 \%$

Conclusions: there were positive demographic and social changes in Niterói. Birth weight was not much affected by prematurity. As in other locations in Brazil, there has been an increase in the number of women undergoing Caesarian sections.

Key words Cesarean section, Birth certificates, Time series studies, Live birth, Birth weight, Prenatal care, Information systems

\section{Resumo}

Objetivos: descrever a tendência temporal de características maternas, assistenciais e dos recémnascidos, em Niterói, cidade do Estado do Rio de Janeiro.

Métodos: estudo ecológico, de tendência temporal, de 2000 a 2009. A fonte de dados foi o Sistema de Informações sobre nascidos vivos (SINASC). Foram descritas variações anuais de: idade materna, escolaridade, história reprodutiva, pré-natal, tipo de parto, cor, peso ao nascer e idade gestacional.

Resultados: a completitude da informação foi alta nos 62.449 nascidos vivos estudados. Reduziram-se os nascimentos e aumentou a primiparidade. Houve redução da gravidez adolescente (2,3\% ao ano) e aumento de mães com mais de 35 anos. Melhorou a escolaridade, com redução do percentual de gestantes com menos de oito anos de estudo. Cerca de $80 \%$ das mulheres realizou sete ou mais consultas de pré-natal. O parto cesáreo apresentou tendência de aumento, realizado por $67,7 \%$ das mulheres em 2009. O baixo peso ao nascer apresentou tendência de queda de 1,1\% ao ano. A prematuridade aumentou de 7,4 para $7,9 \%$.

Conclusões: Niterói apresentou evolução demográfica e social favorável. O peso ao nascer não foi muito afetado pela prematuridade. Como em outros locais do Brasil, tem se elevado o número de cesarianas.

Palavras-chave Cesárea, Declaração de nascimento, Estudos de séries temporais, Nascido vivo, Peso ao nascer, Pré-natal, Sistemas de informação 


\section{Introdução}

A saúde de mulheres e crianças brasileiras teve avanços importantes nas últimas três décadas, consequentes a mudanças sociodemográficas, econômicas e dos sistemas de saúde, principalmente com a implantação do Sistema Único de Saúde (SUS). Ainda há desigualdades regionais e indicadores preocupantes, como a alta taxa de cesarianas e o aumento de nascimentos pré-termo. ${ }^{1}$ Também neste período, foram implantados sistemas de informação sobre estatísticas vitais no país, que contribuíram para o monitoramento destas mudanças. 1,2

O Sistema de Informações sobre Nascidos Vivos (SINASC), implantado no ano de 1990 pelo Ministério da Saúde, tem como base a Declaração de Nascido Vivo (DNV) - documento cuja emissão é obrigatória no serviço de saúde onde ocorreu o parto, por meio do qual se podem obter informações sobre o nascimento, o local de ocorrência, a mãe, a gestação, o parto e o recém-nascido (RN). ${ }^{2}$ Desta forma, constitui-se em instrumento para elaboração de diagnósticos de saúde e avaliação de ações na área materno-infantil. 1,2

O SINASC vem sendo usado para descrever populações de nascidos vivos em várias cidades do Brasil. A revisão de Paiva et al. 3 identificou dez artigos incluindo descrições do perfil de nascidos vivos, no período entre 1994 e 2005, a maioria nos Estados de São Paulo, Minas Gerais e Rio de Janeiro. Um estudo descritivo em Campinas (SP) discorreu sobre os nascidos vivos da cidade em 2005 comparando-os com os nascimentos em 2001.4 Alguns estudos de análise de séries temporais de informações constantes no SINASC também vêm contribuindo para a área de saúde materno infantil.5,6 No estudo sobre a mortalidade infantil em Porto Alegre (RS), também foi analisada a evolução temporal de variáveis do SINASC. 7 Paralelamente, estudos sobre a qualidade dos dados do SINASC vêm demonstrando melhora no preenchimento e na consistência de quase todas as variáveis. ${ }^{8}$

É de fundamental importância a realização de análise dos indicadores de saúde materno-infantil no nível municipal, no cenário de descentralização das ações de saúde.

Em Niterói, cidade do Estado do Rio de Janeiro, apenas um estudo descritivo foi realizado em 1992,9 avaliando informações presentes nas 8789 DNV de crianças residentes na cidade e discutindo os possíveis fatores de risco à saúde infantil. Este estudo destacou o elevado número de cesarianas realizado na cidade e a relação inversa entre baixo peso ao nascer e escolaridade materna. Não há outros estudos publicados sobre a situação maternoinfantil na cidade.

O presente estudo teve como objetivo descrever a tendência temporal dos indicadores sociodemográficos e de saúde de mães e seus recém-nascidos em Niterói, durante a última década. O relato destes indicadores pode fornecer subsídios para análise da situação de saúde materno-infantil e avaliação dos serviços de saúde locais.

\section{Métodos}

Trata-se de um estudo ecológico descritivo de séries temporais de indicadores sociodemográficos e de saúde materno-infantil do município de Niterói, de 2000 a 2009. Em 2010, o município possuía população estimada de 487.562 habitantes, sendo a sexta mais populosa (http://www.ibge.gov.br/cidadesat/ painel/), com o melhor nível de alfabetização e a primeira posição no ranking de Índice de Desenvolvimento Humano (IDH) $(0,886)$ no Estado do Rio de Janeiro (http://www.pnud.org.br/atlas/ tabelas/index.php).

A fonte de dados foi o SINASC, sendo usados os bancos de dados cedidos pela Fundação Municipal de Saúde de Niterói.

As informações da mãe e do RN foram analisadas utilizando-se as seguintes variáveis: idade materna; grau de escolaridade da mãe, número de consultas de pré-natal, história reprodutiva, tipo de gravidez, tipo de parto, sexo, peso ao nascer (analisada de forma contínua e por faixas de peso), idade gestacional, cor/raça. Para as variáveis maternas e assistenciais, as mulheres com partos múltiplos contaram apenas uma vez na totalização dos dados. Para as variáveis do recém-nascido, foram excluídas 56 observações discrepantes, de acordo com um roteiro de crítica do SINASC, desenvolvido pela Secretaria de Saúde e Defesa Civil do Município do Rio de Janeiro, nas quais $\mathrm{RN}$ com peso inferior a $1500 \mathrm{~g}$ estavam registrados com idade gestacional igual ou maior a 37 semanas. Para a variável cor da pele, foram excluídas as categorias com frequência inferior a $1 \%$. A forma de categorização das variáveis obedeceu à disponibilidade das informações do SINASC.

Foram calculadas as medianas, as médias e os desvios-padrão anuais do peso ao nascer e testadas as diferenças estatísticas entre as médias anuais, considerando todo o período (Teste post-hoc Scheffé para múltiplas comparações). Para análise das curvas de distribuição de frequência do peso ao nascer foram criados intervalos a cada $100 \mathrm{~g}$ de peso.

Os dados do banco do SINASC foram proces- 
sados e analisados com o programa SPSS versão 17.0 .

Os indicadores selecionados para a análise de tendência temporal foram aqueles mais associados a desfechos perinatais: a) Características maternas: proporção de mães adolescentes ( $\leq 19$ anos) e de mães com 35 e mais, proporção de mães por categoria de escolaridade (baixa - menor que oito anos e alta - maior ou igual a doze anos), proporção de mães primíparas, proporção de mães com filho morto anteriormente, proporção de mães com gravidez atual múltipla; b) Características assistenciais: proporção de mães que realizaram três ou menos consultas (pré-natal inadequado) e sete ou mais consultas (pré-natal adequado); proporção de nascidos vivos de parto cesáreo; c) Características do recém-nascido: proporção de recém-nascidos por categoria de cor/raça; proporção de nascidos vivos de muito baixo peso $(<1500 \mathrm{~g})$ e de baixo peso $(<2500 \mathrm{~g})$ ao nascer, prematuros ( $<37$ semanas $)$, muito prematuros $(<32$ semana) e de leve a moderada prematuridade (entre 32 a 36 semanas).

A análise da tendência temporal das características maternas e dos nascidos vivos foi feita por meio da análise de regressão de pontos de junção, usando o software Joinpoint Regression Program. 10

Essa análise ajusta uma série de linhas e seus pontos de junção, em uma escala logaritimica, testando as tendências anuais. $\mathrm{O}$ teste de significância é o Monte Carlo Permutation method (que ajusta a melhor linha para cada segmento). Uma vez que estes segmentos são estabelecidos, o percentual anual de mudança (APC) é estimado e testado. Quando há um ponto de junção em que o sentido se inverte, os períodos são analisados separadamente. Nesta situação, o ano final de um período coincide com o ano inicial do próximo na análise e, para cada variável, estes períodos podem ser diferentes em relação aos anos analisados, dependendo do momento em que se detecta a mudança. Também pode haver diferentes padrões de tendência, em períodos de tempo diversos, para as categorias de uma mesma variável. Caso não haja mudança, o período é analisado de forma integral; no caso do estudo, de 2000 a 2009.

Este aplicativo, tradicionalmente usado em estudos epidemiológicos de câncer e de saúde ocupacional, passou a ser incorporado na área maternoinfantil11 e recentemente foi aplicado para avaliar tendência temporal de peso ao nascer no Brasil. 12

Este estudo, obedecendo à Resolução do Conselho Nacional de Saúde (CNS) n 196/96, foi aprovado pelo Comitê de Ética em pesquisa do HUAP, em 17/12/2010 (CEP-CMM/HUAP 317/10;
CAAE 6123.0.000.258-10), não implicando qualquer risco para as puérperas e seus bebês. Os dados foram coletados do banco do SINASC de Niterói de forma confidencial.

\section{Resultados}

Foram incluídos no estudo 62.449 recém-nascidos no período de 2000 a 2009. A incompletitude da informação foi baixa para a maioria das variáveis. Idade e escolaridade da mãe, tipo de parto, número de filhos vivos e peso do bebê tiveram o preenchimento quase completo, com menos ou igual a $0,1 \%$ de ausência. Idade gestacional e número de consultas de pré-natal apresentaram $0,5 \%$ e $1 \%$ de incompletitude, respectivamente. As informações sobre cor/raça do RN e número de filhos mortos estavam ausentes em $1,9 \%$ e $2 \%$, respectivamente. As categorias de cor/raça amarela e indígena somaram apenas 100 informações e não foram apresentadas.

A Tabela 1 mostra o número absoluto de nascidos vivos e os percentuais do ano de 2000 e de 2009. O número absoluto de nascidos vivos sofreu redução no período analisado: 7185 (2000) e 6069 (2009). Também foram observadas mudanças para as variáveis maternas: idade, escolaridade e número de filhos vivos; para as variáveis assistenciais, aumentou o percentual de cesarianas; em relação ao $\mathrm{RN}$, mudaram o percentual de baixo peso e prematuridade (Tabela 1).

Nas Tabelas 2, 3 e 4 são apresentados os percentuais anuais (APC) e características destas mudanças: em que períodos ocorreram e se houve significância estatística.

Em relação às variáveis maternas (Tabela 2), a mudança da maioria das variáveis foi detectada entre 2000 e 2009. Para idade materna, houve redução anual constante de mães adolescentes $(2,3 \%)$ e aumento de gravidezes em mulheres com mais de 35 anos $(2,9 \%)$, ambos estatisticamente significantes.

Também se observou mudança para o nível educacional, com uma redução anual $(p<0,05)$ da baixa escolaridade. Com isto, a categoria predominante mudou, em dez anos, de baixa escolaridade para intermediária. Percebe-se discreto aumento do número de mulheres com mais de 12 anos de estudo, porém sem tendência estatisticamente significante.

Quanto à história reprodutiva materna, considerando o período de 2000 a 2009, houve aumento, com significância estatística, do número de mulheres sem filho vivo anterior. Para o percentual de mulheres com um a dois filhos, ocorreu a diminuição no período de 2000 a 2005 ; depois não houve e não perdeu magnitude e significância 
Número absoluto e percentual de nascidos vivos segundo características maternas, assistenciais e neonatais. Niterói, 2000 e 2009.*

\begin{tabular}{|c|c|c|c|c|}
\hline \multirow[t]{2}{*}{ Variáveis } & \multicolumn{2}{|c|}{ Ano $2000(\mathrm{~N}=7185)$} & \multicolumn{2}{|c|}{ Ano $2009(\mathrm{~N}=6069)$} \\
\hline & $\mathrm{n}$ & $\%$ & $\mathrm{n}$ & $\%$ \\
\hline \multicolumn{5}{|l|}{ Idade materna (anos) } \\
\hline$\leq 19$ & 1347 & 18,8 & 871 & 14,4 \\
\hline $20-34$ & 4983 & 69,4 & 4329 & 71,3 \\
\hline$\geq 35$ & 846 & 11,8 & 869 & 14,3 \\
\hline \multicolumn{5}{|l|}{ Escolaridade materna (anos) } \\
\hline$<8$ & 2750 & 38,8 & 1443 & 23,9 \\
\hline $8-11$ & 2176 & 30,7 & 2578 & 42,7 \\
\hline$\geq 12$ & 2161 & 30,5 & 2017 & 33,4 \\
\hline \multicolumn{5}{|l|}{ Filhos vivos } \\
\hline Nenhum & 3487 & 48,6 & 3284 & 54,2 \\
\hline 1 a 2 & 3157 & 44,0 & 2424 & 40,0 \\
\hline$\geq 3$ & 531 & 7,4 & 345 & 5,7 \\
\hline \multicolumn{5}{|l|}{ Filhos mortos } \\
\hline Nenhum & 6505 & 92,4 & 5524 & 93,0 \\
\hline$\geq 1$ & 535 & 7,6 & 545 & 7,0 \\
\hline \multicolumn{5}{|l|}{ Consultas de pré-natal } \\
\hline$\leq 3$ & 417 & 5,8 & 121 & 3,7 \\
\hline $4-6$ & 920 & 12,9 & 889 & 14,9 \\
\hline$\geq 7$ & 5797 & 81,3 & 4850 & 81,4 \\
\hline \multicolumn{5}{|l|}{ Tipo de Parto } \\
\hline Cesariana & 4390 & 61,1 & 4108 & 67,7 \\
\hline \multicolumn{5}{|l|}{ Sexo } \\
\hline Masculino & 3647 & 50,8 & 3187 & 52,6 \\
\hline \multicolumn{5}{|l|}{ Cor da pele } \\
\hline Branca & 4575 & 63,8 & 3847 & 64,8 \\
\hline Preta & 294 & 4,0 & 305 & 5,1 \\
\hline Parda & 2293 & 32,2 & 1782 & 30,1 \\
\hline \multicolumn{5}{|l|}{ Peso ao nascer } \\
\hline Baixo peso $(<2500 \mathrm{~g})$ & 632 & 8,8 & 509 & 8,4 \\
\hline \multicolumn{5}{|l|}{ Idade gestacional } \\
\hline Pré-termo (<37 semanas) & 529 & 7,4 & 477 & 7,9 \\
\hline
\end{tabular}

* Fonte: SINASC. O valor do $\mathrm{N}$ para cada categoria expressa o número de declarações estudadas e variou de acordo com a completitude dos dados.

estatística. Já para as mulheres com três ou mais filhos, o percentual declinou em todo o período. Por outro lado, não se observou redução estatisticamente significativa do percentual de mulheres que tiveram filho morto anteriormente. Por último, a primiparidade teve tendência estatisticamente significante de aumento anual $(1,8 \%)$

A proporção de nascidos vivos de gravidez múltipla (dois ou mais bebês) teve um incremento com tendência anual de aumento de $2,1 \%(p<0,05)$.

$\mathrm{Na}$ Tabela 3, observa-se que a proporção de recém-nascidos de mães com menos de três consultas de pré-natal reduziu apenas no período de 2000 a 2003 sendo observada posteriormente, uma tendência crescente até 2009, ambos sem significância estatística.

Já o percentual de parto cesáreo apresentou aumento anual de $1,1 \%(p<0,05)$, sendo a via de parto utilizada por quase $70 \%$ em 2009 . Neste ano, em mulheres com baixa escolaridade o percentual de cesarianas foi de $42 \%$, e atingiu $89 \%$ daquelas com mais de 12 anos de estudo (dados não mostrados na Tabela). 
Tabela 2

Percentuais de mudança anual (APC) das características maternas sociodemográficas e reprodutivas.

\begin{tabular}{|c|c|c|c|}
\hline \multirow[t]{3}{*}{ Variáveis } & Tendência 1 ** & Tendência $2 * *$ & Tendência 3 * * \\
\hline & APC (IC95\%) & APC (IC95\%) & APC (IC95\%) \\
\hline & $2000-2005$ & $2005-2009$ & $2000-2009$ \\
\hline \multicolumn{4}{|c|}{ Idade materna (anos) } \\
\hline Até 19 & - & - & $-2,3 *(-3,5 ;-1,0)$ \\
\hline$\geq 35$ & - & - & $2,9 *(2,3 ; 3,5)$ \\
\hline \multicolumn{4}{|c|}{ Escolaridade materna (anos) } \\
\hline$<8$ & - & - & $-5,1 *(-6,5 ;-3,7)$ \\
\hline $8-11$ & - & - & $4,0 *(3,0 ; 5,1)$ \\
\hline$\geq 12$ & - & - & $0,6(-1,4 ; 2,5)$ \\
\hline Primiparidade & - & - & $1,8 *(1,4 ; 2,2)$ \\
\hline \multicolumn{4}{|l|}{$\mathrm{N}^{\circ}$ filhos vivos } \\
\hline Nenhum & - & - & $1,5^{*}(1,2 ; 1,8)$ \\
\hline $1-2$ & $-2,1 *(-2,8 ;-1,4)$ & $-0,2(-1,3 ; 0,9)$ & \\
\hline$\geq 3$ & - & - & $-3,0 *(-4,5 ;-1,6)$ \\
\hline \multicolumn{4}{|c|}{$\mathrm{N}^{\circ}$ de filhos mortos } \\
\hline$\geq 1$ & - & - & $-2,9(-6,3 ; 0,6)$ \\
\hline \multicolumn{4}{|c|}{ Tipo de gravidez } \\
\hline Múltipla & - & - & $2,1 *(0,3 ; 3,9)$ \\
\hline
\end{tabular}

Fonte SINASC: análise por regressão joinpoint dos nascidos vivos de Niterói, 2000-2009; * $p<0,05 ; *$ * os períodos onde foi detectada tendência podem diferir, de acordo com a variável estudada.

\section{Tabela 3}

Percentuais de mudança anual (APC) do número de consultas de pré-natal e tipo de parto.

\begin{tabular}{lccc}
\hline Variáveis & Tendência 1** & Tendência 2** & Tendência $3 * *$ \\
\cline { 2 - 4 } & $\begin{array}{c}\text { APC (IC95\%) } \\
2000-2003\end{array}$ & $\begin{array}{c}\text { APC (IC95\%) } \\
2003-2009\end{array}$ & $\begin{array}{c}\text { APC (IC95\%) } \\
2000-2009\end{array}$ \\
\hline $\begin{array}{l}\text { Consultas de pré-natal } \\
\leq 3\end{array}$ & $-15,6(-32,4 ; 5,2)$ & $3,5(-3,0 ; 10,4)$ & \\
$\geq 7$ & - & - & $-0,1(-0,7 ; 0,4)$ \\
$\begin{array}{l}\text { Tipo de parto } \\
\text { Cesáreo }\end{array}$ & - & - & $1,1 *(1,0 ; 1,2)$ \\
\hline
\end{tabular}

Fonte SINASC: análise por regressão joinpoint dos nascidos vivos de Niterói, 2000-2009; * $p<0,05$; * os períodos onde foi detectada tendência podem diferir, de acordo com a variável estudada. 
Percentuais de mudança anual (APC) das características dos nascidos vivos.

\begin{tabular}{|c|c|c|c|}
\hline \multirow[t]{3}{*}{ Variáveis } & Tendência 1 ** & Tendência 2 * * & Tendência 3 ** \\
\hline & APC $(I C 95 \%)$ & APC $(I C 95 \%)$ & APC $(I C 95 \%)$ \\
\hline & & & 2000-2009 \\
\hline Sexo Masculino & - & - & $-0,1(-0,3 ; 0,2)$ \\
\hline \multicolumn{4}{|l|}{ Peso ao nascer ( $\mathrm{g}$ ) } \\
\hline$<2500$ & - & - & $-1,1(-1,8 ;-0,5)$ \\
\hline$<1500$ & - & - & $-0,07(-1,3 ; 1,2)$ \\
\hline Idade gestacional (semanas) & $2000-2004 * *$ & $2004-2009 * *$ & \\
\hline$<32$ & $6,1 *(3,7 ; 8,5)$ & $-1,7 *(-3,1 ;-0,2)$ & \\
\hline $32-36$ & $4,7 *(1,0 ; 8,4)$ & $-3.4^{*}(-5,7 ;-1,1)$ & \\
\hline$<37$ & $4,8 *(1,7 ; 7,9)$ & $-3,1 *(-5,0 ;-1,2)$ & \\
\hline Cor/Raça & $2000-2007$ * * & $2007-2009 * *$ & \\
\hline Branca & $-0,7 *(-1,1 ;-0,3)$ & $3,1(-0,7 ; 7,0)$ & \\
\hline \multirow[t]{2}{*}{ Preta } & - & - & $8,7(-0,9 ; 19,2)$ \\
\hline & $2000-2002 * *$ & $2002-2009 * *$ & \\
\hline Parda & $6,5(-9,0 ; 24,6)$ & $-1,9 *(-3,5 ;-0,2)$ & \\
\hline
\end{tabular}

Fonte SINASC: análise por regressão joinpoint dos nascidos vivos de Niterói, 2000-2009; * $p<0,05 ;$ ** períodos diferentes conforme a variável analisada.

Na Tabela 4, sobre as características dos RN, houve também diferentes padrões de tendência.

Não se observou mudança no percentual de bebês do sexo masculino, ao longo de todo o período. Em relação ao peso, o baixo peso ao nascer (BPN) apresentou uma tendência à diminuição, de $1,1 \%$ ao ano, enquanto a proporção de muito baixo peso não apresentou mudanças no período.

$\mathrm{Na}$ Figura 1, mostram-se as curvas de distribuição de frequência dos nascidos vivos segundo peso ao nascer e respectivas médias, do primeiro e do último ano analisados. O aumento das médias não foi acompanhado de uma assimetria da curva à direita em 2009. As medianas do peso ao nascer aumentaram de $3190 \mathrm{~g}$ em 2000 para $3205 \mathrm{~g}$ em 2009. O discreto aumento das médias do peso ao nascer, considerando-se todos os anos analisados (resultados não apresentados), foi estatisticamente significante $(p<0,001)$.

Quanto à idade gestacional, houve diferentes comportamentos nos períodos de 2000 a 2004 e 2004 a 2009. Na análise de tendência, para todos os intervalos de prematuridade, o incremento anual se deu na primeira metade do período (2000 a 2004), apresentando depois tendência de reversão, ambos estatisticamente significantes, porém a velocidade de reversão foi menor que a velocidade de aumento e, em 2009, o percentual alcançou 7,9\% (Tabela 4).
Deve ser ressaltado que o maior incremento anual foi para os menores de 32 semanas de gestação $(6,1 \%)$.

Para a variável cor/raça, considerando os $\mathrm{RN}$ brancos e pardos, as tendências observadas nos períodos de 2000 a 2007 foram respectivamente diminuição discreta e aumento, este sem significância estatística. De 2007 a 2009, houve diminuição dos pardos. Para a cor preta, a mudança, embora detectada para todo o período, não alcançou significância estatística.

\section{Discussão}

A análise mostrou ter havido algumas mudanças das características maternas, e as relacionadas ao parto, gestação e recém-nascidos no município de Niterói nos últimos dez anos.

Em relação às características maternas, quase todas tiveram mudanças que ocorreram no período de 2000 a 2009, de forma constante.

Constatou-se ter havido redução constante do percentual de mães adolescentes em Niterói. Esse resultado é semelhante ao observado no município de Campinas, no Estado de São Paulo, entre 2001 e 20054 e ao de Porto Alegre, Rio Grande do Sul, entre 1996 e 2008.7 Esta mudança é benéfica para a população materno-infantil, já que a literatura tem mostrado que a gravidez na adolescência está geral- 


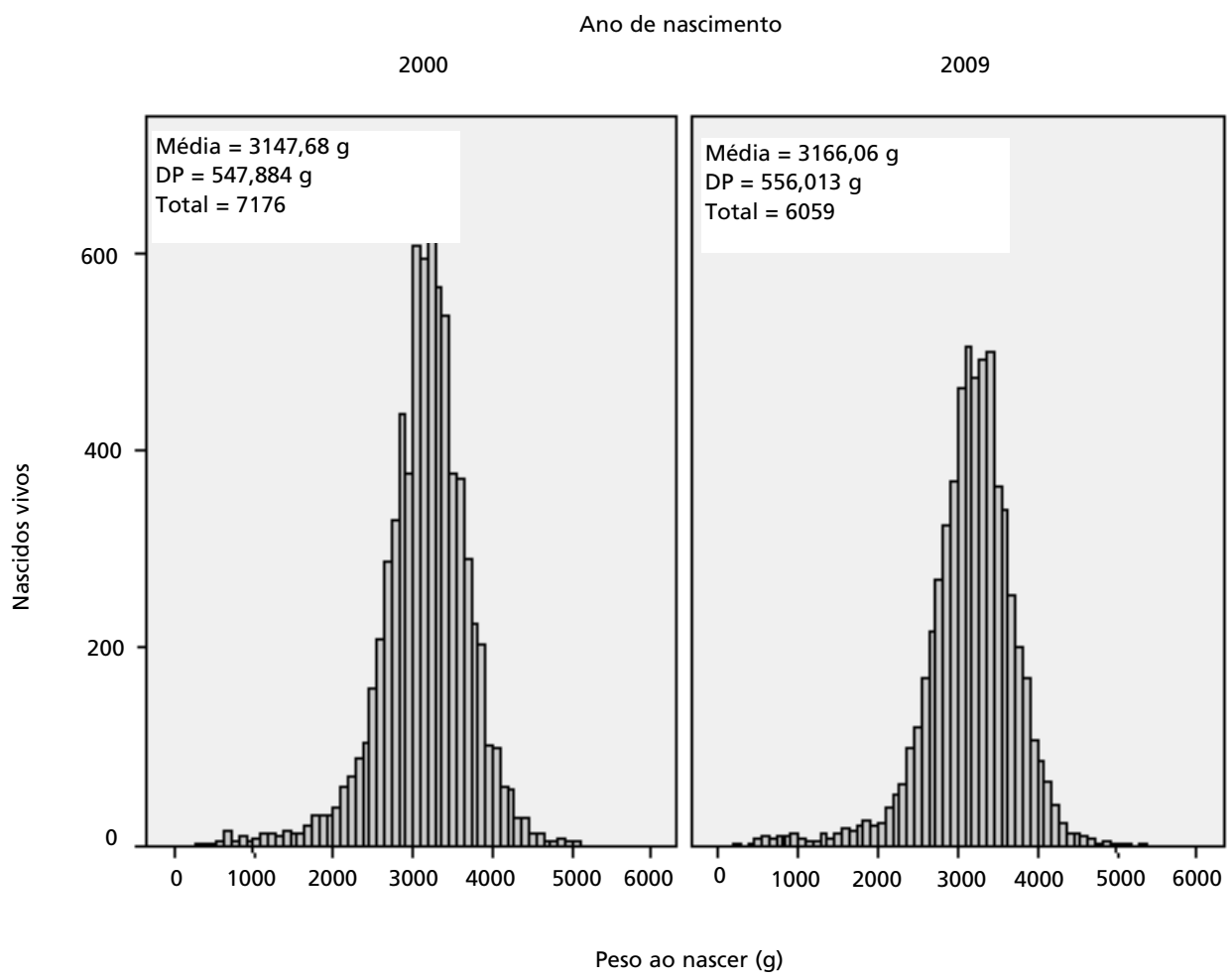

*Diferença de média do peso - Teste post-hoc Scheffé para múltiplas comparações (considerando todos os anos do período). Aqui estão apresentados somente os anos 2000 e $2009(p<0,001)$.

mente associada a desfechos perinatais e infantis mais graves. 13,14

Ainda em relação à idade materna, houve um aumento constante da gravidez em mulheres com mais de 35 anos. Esse resultado é semelhante aos de outras cidades com índice elevado de desenvolvimento, como Porto Alegre e Campinas. ${ }^{4,7}$ Embora seja uma mudança etária que geralmente acompanha o desenvolvimento, deve-se atentar para o maior risco de desfechos maternos e perinatais desfavoráveis. 15,16

Quanto ao grau de instrução das mães, as mudanças foram todos favoráveis, com queda do percentual de baixa escolaridade e aumento do nível intermediário e superior (embora este último sem significância estatística). Em Porto Alegre, o percentual de mulheres com baixa escolaridade também caiu e o de mulheres com doze anos ou mais de estudo aumentou. ${ }^{7}$ Em Campinas, os resultados foram semelhantes. ${ }^{4}$ Esta mudança em Niterói é relevante, pois a escolaridade materna elevada tem se mostrado um forte fator protetor para vários desfe- chos da saúde da mulher e da criança, destacando-se a mortalidade infantil. 17

Observou-se um aumento discreto e constante dos partos múltiplos. Este resultado também foi observado em Porto Alegre, no período de 1995 a 200418 e em vários países desenvolvidos nos últimos anos. ${ }^{19}$ Especula-se que este aumento se deva à maior frequência de grávidas com mais de 35 anos e aos tratamentos de infertilidade. 19

A queda da fecundidade foi observada em Niterói, com a redução do número de nascidos vivos e do número de filhos de cada mulher semelhante a Campinas ${ }^{4}$ e Porto Alegre. ${ }^{7}$ Quanto aos filhos nascidos mortos, cerca de 7\% das mães informou a ocorrência anterior de perda fetal e este percentual não mudou ao longo do período. Estudos específicos sobre mortalidade fetal devem ser realizados para entender melhor o fenômeno na cidade.

A maioria das mulheres realizou sete ou mais consultas de pré-natal em todo o período estudado, não se observando mudanças do percentual. É necessário avaliar futuramente a qualidade do pré- 
natal20 e possíveis desigualdades no acesso, relatados em outros estudos. 21,22

Destaca-se o aumento da frequência do parto cesáreo no período de estudo. Essa tendência preocupante de aumento de cesarianas também foi observada em Porto Alegre e em Campinas. ${ }^{4,7}$ Em 1992, no município de Niterói havia predomínio do parto vaginal apenas entre mulheres com baixo grau de instrução (52\%), sendo a cesariana a via de parto mais frequente entre as mulheres com nível de escolaridade mais elevado (59\%). ${ }^{9}$ Em 2009, o valor foi semelhante nas mulheres com menor escolaridade, mas chegou a $89 \%$ naquelas com mais de doze anos de estudo. Esses dados estão de acordo com o estudo de coorte realizado no município de Pelotas, Rio Grande do Sul, em 2004, no qual a maior frequência do parto cesáreo estava fortemente associada à maior escolaridade e ao parto em unidades privadas. 23

$\mathrm{O}$ peso ao nascer apresentou uma discreta tendência de aumento, verificada na mediana, na média e de queda na proporção do BPN. Este achado é discordante dos demais estudos em locais desenvolvidos do Brasil, onde se observa estabilidade ou mesmo aumento do percentual de baixo peso ao nascer. 11,24 Deve ser ressaltado que, segundo estes estudos, o aumento tem sido observado nas mulheres de melhor condição socioeconômica e tem sido associado ao aumento dos nascimentos pré-termo. Não se observou aumento do percentual de bebês de muito baixo peso em Niterói, diferentemente do relatado em Porto Alegre. 25

Quanto à idade gestacional, observou-se um aumento de partos prematuros, concentrado no período de 2000 a 2004, que não se manteve a seguir e deve ser monitorado, já que outros estudos brasileiros apontam essa tendência de aumento na prematuridade nos últimos anos.4,7 Os estudos de Silveira et al., 26,27 usando diferentes tipos de informação, mostram discordância entre as prevalências de partos pré-termo - menores quando o SINASC é a fonte e maiores nos estudos com dados primários. No entanto, a tendência de aumento da prematuridade no país é corroborada em praticamente todos os estudos.

O tempo relativamente curto da série temporal dez anos - foi uma das limitações do estudo, mas optou-se por trabalhar com um período em que a DNV já estava totalmente implantada sem grandes alterações na forma de coleta das variáveis. A completitude dos dados foi muito boa, mas sua consistência ainda não foi estudada em Niterói. No entanto, como algumas variáveis já foram bem estudadas quanto à confiabilidade e validade em outras localidades brasileiras, acredita-se que não tenha havido comprometimento do estudo.

Os achados ratificam o status de Niterói como cidade desenvolvida, quanto às características demográficas e sociais: queda da fecundidade, aumento da idade materna e aumento da escolaridade. No entanto, ao contrário de outras cidades com estas características, o peso ao nascer não tem sido tão afetado pela prematuridade crescente. Uma provável explicação é a queda do crescimento intrauterino restrito (CIUR), conforme proposto por Barros et al. ${ }^{28}$ Esta variável não pôde ser avaliada no período, pela forma categorizada da idade gestacional no SINASC. Futuramente, com a implantação da nova DNV, será possível aprofundar a pesquisa sobre prematuridade e CIUR, já que a variável passou a ser registrada em semanas completas.

Chama a atenção o nível já elevado e ainda crescente de cesarianas. Esta "igualdade" com outras cidades desenvolvidas do Brasil não é desejável. A explicação para o aumento de cesarianas no país pode estar relacionada ao modelo obstétrico vigente, altamente medicalizado e com grande uso de tecnologias. 29

O atual estudo demonstrou que é possível analisar mudanças de base populacional na área de saúde materno-infantil, usando o SINASC. Deve haver investimento continuado para manter a completitude dos dados e realização de avaliações de sua confiabilidade, para dar mais respaldo às pesquisas.

Os resultados apontaram a necessidade de monitorar o aumento de cesarianas em Niterói assim como o percentual de prematuros. O uso de séries temporais se mostra bastante útil para este acompanhamento e abre perspectivas de novas análises de dados secundários.

\section{Agradecimentos}

Aos profissionais do Observatório de Saúde da Fundação Municipal de Saúde de Niterói: Maria Clara Gomes Ferraz, Mônica Santos Stavola e Sylvio Rodrigues Torres Filho.

Suelem do Rozario teve bolsa de Mestrado do Conselho Nacional de Desenvolvimento Científico e Tecnológico $(\mathrm{CNPq})$ durante a realização deste trabalho. 


\section{Referências}

1. Victora CG, Aquino EM, Leal MC, Monteiro CA, Barros FC, Szwarcwald CL. Saúde de mães e crianças no Brasil: progressos e desafios. Lancet. 2011; 377: 1863-76.

2. Mello-Jorge MHP, Laurenti R, Gotlieb SLD. Análise da qualidade das estatísticas vitais brasileiras: a experiência de implantação do SIM e do SINASC. Ciên Saúde Colet (Rio J.). 2007; 12: 643-54.

3. Paiva NS, Coeli CM, Moreno AB, Guimaraes RM, Camargo KR. Sistema de informações sobre nascidos vivos: um estudo de revisão. Ciên Saúde Colet. 2011; 16 (Supl. 1): S1211-20.

4. Morcillo AM, Carniel EF, Zanolli ML, Moreno LZ, Antonio MARGM. Caracterização das mães, partos e recém-nascidos em Campinas, São Paulo, 2001 e 2005. Rev Paul Pediatr. 2010; 28: 269-75.

5. Gibson, G, Costa LS, Koifman S. Time trend of the male proportion at birth in Brazil, 1979-2004. Int J Environ Res Public Health. 2009; 6: 2193-204.

6. Gouveia N, Bremner SA, Novaes HMD. Association between ambient air pollution and birth weight in São Paulo, Brazil. J Epidemiol Community Health. 2004; 58: 11-7.

7. Hernandez AR, Silva CH, Agranonik M, Quadros FM, Goldani MZ. Tendências das taxas de mortalidade infantil e de seus fatores de risco. Cad Saúde Pública. 2011; 27: 2188-96.

8. Romero ED, Cunha CB. Avaliação da qualidade das variáveis epidemiológicas e demográficas do sistema de informações sobre nascidos vivos, 2002. Cad Saúde Pública. 2007; 23: 701-14

9. Kale PL. Primeira avaliação do sistema de informações sobre nascidos vivos no município de Niterói. Cad Saúde Colet (Rio J.). 1997; 5: 53-64.

10. National Cancer Institute. Joinpoint Regression Program, Version 3 - April 2011; Statistical Methodology and Applications Branch and Data Modeling Branch, Surveillance Research Program National Cancer Institute. [acesso em fev 2012]. Disponível em: http://surveillance.cancer.gov/joinpoint/

11. Wingo PA, Smith RA, Tevendale HD, Ferré C. Recent changes in the trends of teen birth rates, 1981-2006. J Adolesc Health. 2011; 48: 281-8.

12. Silva AAM, Silva LM, Barbieri MA, Bettiol H, Carvalho LM, Ribeiro VS, Goldani MZ. The epidemiologic paradox of low birth weight in Brazil. Rev Saúde Pública. 2010; 44: 767-75.

13. Chen XK, Wen SW, Fleming N, Demissie K, Rhoads GG, Walker M. Teenage pregnancy and adverse birth outcomes: a large population based retrospective cohort study. Int $\mathrm{J}$ Epidemiol. 2007; 36: 368-73

14. Oliveira EFV, Gama SGN, Silva CMFP. Gravidez na adolescência e outros fatores de risco para mortalidade fetal e infantil no município do Rio de Janeiro, Brasil. Cad Saúde Pública. 2010; 26: 576-8.
15. Fonseca SC, Coutinho ES. Mortalidade perinatal no Brasil: revisão da metodologia e dos resultados. Cad Saúde Pública. 2004; 20 (Supl. 1): S7-19.

16. Morse ML, Fonseca SC, Barbosa MD, Calil, MB, Eyer FPC. Mortalidade materna no Brasil: o que mostra a produção científica nos últimos 30 anos? Cad Saúde Pública. 2011; 27: 623-38

17. Garcia LP, Santana LR. Evolução das desigualdades socioeconômicas na mortalidade infantil no Brasil, 1993-2008. Ciênc Saúde Colet (Rio J.). 2011; 16: 3717-28.

18. Homrich da Silva C, Goldani MZ, Moura Silva AA, Agranonik M, Bettiol H, Barbieri MA, Rona R. The rise of multiple births in Brazil. Acta Paediatr. 2008; 97: 1019-23.

19. Blondel B, Kaminski M. Trends in the occurrence, determinants, and consequences of multiple births. Semin Perinatol. 2002; 26: 239-49.

20. Andreucci CB, Cecatti JG. Desempenho de indicadores de processo do Programa de Humanização do Pré-natal e Nascimento no Brasil: uma revisão sistemática. Cad Saúde Pública. 2011; 27: 1053-64.

21. Almeida SDM, Barros MBA. Equidade e atenção à saúde da gestante em Campinas (SP), Brasil. Rev Panam Salud Publica. 2005; 17: 15-25.

22. Cesar JA, Mano PS, Carlotto K, Gonzalez-Chica DA, Mendoza-Sassi RA. Público versus privado: avaliando a assistência à gestação e ao parto no extremo sul do Brasil. Rev Bras Saúde Matern Infant. 2011; 11: 257-63.

23. Barros AJ, Santos IS, Matijasevich A, Domingues MR, Silveira M, Barros FC, Victora CG. Patterns of deliveries in a Brazilian birth cohort: almost universal cesarean sections for the better-off. Rev Saúde Pública. 2011; 45: 635-43.

24. Andrade CLT, Szwarcwald CL, Castilho EA. Baixo peso ao nascer no Brasil de acordo com as informações sobre nascidos vivos do Ministério da Saúde, 2005. Cad Saúde Pública. 2008; 24: 2564-72.

25. Silva CH, Agranonik M, Silva AA, Bettiol H, Barbieri MA, Goldani MZ. Secular trend of very low birth weight rate in Porto Alegre, Southern Brazil. J Biosoc Sci. 2010; 42: $243-$ 53.

26. Silveira MF, Santos IS, Barros AJD, Matijasevich A, Barros FC, Victora CG. Aumento da prematuridade no Brasil: revisão de estudos de base populacional. Rev Saúde Pública. 2008; 42: 957-64.

27. Silveira MF, Santos IS, Matijasevich A, Malta DC, Duarte EC. Nascimentos pré-termo no Brasil entre 1994 e 2005 conforme o Sistema de Informações sobre Nascidos Vivos (SINASC). Cad Saúde Pública. 2009; 25: 1267-75.

28. Barros FC, Victora CG, Matijasevich A, Santos IS, Horta BL, Silveira MF, Barros AJ. Preterm births, low birth weight, and intrauterine growth restriction in three birth cohorts in Southern Brazil: 1982, 1993 and 2004. Cad Saúde Pública. 2008; 24 (Suppl. 3): S390-8.

29. Patah LEM, Malik AM. Modelos de assistência ao parto e taxa de cesárea em diferentes países. Rev Saúde Pública. 2011; 45: 185-94 
30. Felisberto E, Frias PG. Novos cenários para os sistemas de informações de eventos vitais à luz da descentralização do sistema de saúde brasileiro. Rev Bras Saúde Matern Infant. $2011 ; 11: 357-8$

Recebido em 7 de maio de 2012

Versão final apresentada em 7 de março 2013

Aprovado em 9 de abril de 2013 